\title{
PHYSICAL PROPERTIES AND MORPHOLOGY OF A NEWLY IDENTIFIED COMPACT $z=4.04$ LENSED SUBMILLIMETER GALAXY IN ABELL 2218
}

\author{
Kirsten K. Knudsen ${ }^{1}$, Jean-Paul Kneib ${ }^{2,3}$, Johan Richard ${ }^{3,4}$, Glen Petitpas $^{5}$, and Eitchi Egami ${ }^{6}$ \\ ${ }^{1}$ Argelander Institut für Astronomie, Auf dem Hügel 71, D-53121 Bonn, Germany; knudsen@ astro.uni-bonn.de \\ ${ }^{2}$ Laboratoire d'Astrophysique de Marseille, OAMP, Université, Aix-Marseilles \& CNRS, 38 rue Frédéric Joliot Curie, 13388 Marseille cedex 13, France \\ ${ }^{3}$ California Institute of Technology, MS 105-24, Pasadena, CA 91125, USA \\ ${ }^{4}$ Physics and Astronomy Department, Durham University, South Road, Durham DH3 1LE, UK \\ ${ }^{5}$ Harvard-Smithsonian Center for Astrophysics, 60 Garden Street, Cambridge, MA 02138, USA \\ ${ }^{6}$ Steward Observatory, University of Arizona, 933 North Cherry Avenue, Tuscon, AZ 85721, USA \\ Received 2009 January 7; accepted 2009 November 9; published 2009 December 30
}

\begin{abstract}
We present the identification of a bright submillimeter (submm) source, SMM J163555.5+661300, detected in the lensing cluster Abell 2218, for which we have accurately determined the position using observations from the Submillimeter Array (SMA). The identified optical counterpart has a spectroscopic redshift of $z=4.044 \pm 0.001$ if we attribute the single emission line detected at $\lambda=6140 \AA$ to $L y \alpha$. This redshift identification is in good agreement with the optical/near-infrared photometric redshift as well as the submm flux ratio $S_{450} / S_{850} \sim 1.6$, the radio-submm flux ratio $S_{1.4} / S_{850}<0.004$, and the $24 \mu \mathrm{m}$ to $850 \mu \mathrm{m}$ flux ratio $S_{24} / S_{850}<0.005$. Correcting for the gravitational lensing amplification of $\sim 5.5$, we find that the source has a far-infrared luminosity of $1.3 \times 10^{12} L_{\odot}$, which implies a star formation rate (SFR) of $230 M_{\odot} \mathrm{yr}^{-1}$. This makes it the lowest-luminosity submillimeter galaxy (SMG) known at $z>4$ to date. Previous $\mathrm{CO}(4-3)$ emission line observations yielded a non-detection, for which we derived an upper limit of the $\mathrm{CO}$ line luminosity of $L_{\mathrm{CO}}^{\prime}=0.3 \times 10^{10} \mathrm{~K} \mathrm{~km} \mathrm{~s}^{-1} \mathrm{pc}^{-2}$, which is not inconsistent with the $L_{\mathrm{CO}}^{\prime}-L_{\mathrm{FIR}}$ relation for starburst galaxies. The best-fit model to the optical and near-infrared photometry give a stellar population with an age of $1.4 \mathrm{Gyr}$ and a stellar mass of $1.6 \times 10^{10} M_{\odot}$. The optical morphology is compact and in the source plane the galaxy has an extent of $\sim 6 \times 3 \mathrm{kpc}$ with individual star-forming knots of $<500 \mathrm{pc}$ in size. J163556 is not resolved in the SMA data, and we place a strict upper limit on the size of the starburst region of $8 \mathrm{kpc} \times 3 \mathrm{kpc}$, which implies a lower limit on the SFR surface density of $12 M_{\odot} \mathrm{yr}^{-1} \mathrm{kpc}^{2}$. The redshift of J163556 extends the redshift distribution of faint, lensed SMGs, and we find no evidence that these have a different redshift distribution than bright SMGs.
\end{abstract}

Key words: galaxies: high-redshift - galaxies: individual (SMM J163556+661300) - submillimeter: galaxies

Online-only material: color figures

\section{INTRODUCTION}

The redshift distribution of submillimeter galaxies (SMGs) is vital to our understanding of the nature of these galaxies and their role in the general picture of galaxy formation and evolution. The first systematic identification and redshift determination of SMGs relied on the radio counterparts and was carried out by Chapman et al. $(2003,2005)$. While the radio and optical/near-infrared counterparts have much better determined positions compared to the original $15^{\prime \prime}$ resolution submillimeter (submm) observations and thus is an efficient approach for identifying the underlying galaxy, the radio emission rapidly dims with the distance and thus is likely to introduce a bias against SMGs with redshift $z>3.5$. Due to the negative $K$-correction, (sub)mm observations offer a great opportunity to search for galaxies beyond $z>4$, and one would expect to find SMGs up to redshifts $z \sim 6-7$. Through modeling of the SMG population Chapman et al. (2005) predicted that about $10 \%$ of the population is at redshift $z \geqslant 4$. Wall et al. (2008) suggested a bimodal redshift distribution, where the brightest SMGs dominate at the highest redshifts and the fainter SMGs dominate toward lower redshifts, as initially proposed by Ivison et al. (2002), equivalent to "cosmic down-sizing."

To date, there has been no systematic samples of very high redshift $(z>3.5)$ SMGs; however, Dannerbauer et al. $(2002,2004)$ and Younger et al. (2007) have presented several high-redshift candidates that were identified through (sub)mm interferometry (with and without radio counterparts). Three
SMGs with accurate redshifts are COSMOS J100054+023436 $(z=4.547$; Capak et al. 2008; Schinnerer et al. 2008), and GN20 and GN20.2 (both $z=4.05$; Daddi et al. 2009b). These three sources have $\mathrm{CO}$ redshifts providing solid evidence for correct identification. The galaxy GOODS 850-5 (also known as GN10) does not have an optical spectroscopic redshift, but based on photometric redshifts two independent groups conclude that it is $z>4$, possibly $z \sim 6$, (Dannerbauer et al. 2008; Wang et al. 2007, 2009). In a recent paper, Daddi et al. (2009a) presented a redshift based on a single millimeter wavelength emission line, which they interpret as the $\mathrm{CO}(4-3)$ line placing the galaxy at redshift $z=4.0424$. Very recently, the highest redshift SMG known to date has been identified, namely the $z=4.76$ galaxy LESSJ033229.4-275619 in the LABOCA map of E-CDF-S (Coppin et al. 2009).

In this paper, we present the identification of the optical counterpart of the submm source SMMJ163555.5+661300 (henceforth J163556), which was detected in the deep Submillimetre Common-User Bolometer Array (SCUBA) ${ }^{7}$ map of Abell 2218 (Kneib et al. 2004; Knudsen et al. 2006) with a lensed flux of $S_{850}=11.3 \mathrm{mJy}$. It is important to note that this is a $10 \sigma$ SCUBA detection, rendering it a very solid detection and not a spurious source. Exploiting the strong gravitational lensing effect by the cluster potential, these SCUBA observations have yielded the largest number of faint submm galaxies detected in one field.

\footnotetext{
7 Submillimetre Common-User Bolometer Array (Holland et al. 1999) mounted at the James Clerk Maxwell Telescope (JCMT).
} 
Here we present multiwavelength follow-up and spectroscopic observations.

Throughout we will assume an $\Omega=0.3, \Lambda=0.7$ cosmology with $H_{0}=70 \mathrm{~km} \mathrm{~s}^{-1} \mathrm{Mpc}^{-1}$. With this cosmology, at $z=4$, $1^{\prime \prime}$ corresponds to $6.95 \mathrm{kpc}$.

\section{OBSERVATIONS}

\subsection{Submillimeter Interferometric Observations}

SMMJ163556 was observed at the Submillimeter Array (SMA; Ho et al. 2004) on Mauna Kea in compact configuration on UTC 2009 May 5 using 7 of the 8 SMA antennas. The resulting image created has $u v$-coverage from $13.7 \mathrm{k} \lambda$ to $121.6 \mathrm{k} \lambda$ at the observed local oscillator frequency of $340 \mathrm{GHz}$. Observations were made in very dry conditions, with $\tau_{225}$ ranging from 0.04 to 0.06 . The full $4 \mathrm{GHz}(2 \mathrm{GHz}$ in each sideband separated by $10 \mathrm{GHz}$ ) were combined to achieve a total rms noise of $1.9 \mathrm{mJy}$ in the final map.

The SMA data were calibrated using the MIR software package developed at Caltech and modified for the SMA. Gain calibration was performed using the nearby quasars $1642+689$, $1638+573$, and 1849+670. Absolute flux calibration was performed using real-time measurements of the system temperatures, with observations of Callisto, Ganymede, Uranus, and Neptune to set the scale. Bandpass calibration was done using Callisto. The data were imaged using Miriad (Sault et al. 1995), where the resulting synthesized beam is $2^{\prime \prime} .1 \times 1$.'.5 with a position angle, P.A. $=39^{\circ}$. To verify the astrometry, the position of $1638+573$ was calibrated using $1642+689$ as reference sources; both calibrators have accurately known positions known from the International Celestial Reference Frame. A fit to the $u v$-data of $1638+573$ shows that it deviates from the known radio position by $\Delta$ R.A. $=00^{\prime} 14\left(00^{\prime} 10\right)$ and $\Delta$ decl. $=0{ }^{\prime} 02\left(0{ }^{\prime} 005\right)$ in the lower (and upper) sideband. As the distance between 1638+573 and $1642+689$ is 11.6 , four times larger than that between $1642+689$ and J163556, the fit provides a conservative upper limit to the systematics uncertainties in the SMA astrometry.

\subsection{Radio Observations}

Radio maps of A2218 at $1.4 \mathrm{GHz}$ and $8.2 \mathrm{GHz}$ were presented by Garrett et al. (2005). The depth of the $1.4 \mathrm{GHz}$ data, which were obtained with the Westerbork Synthesis Radio Telescope (WSRT), is $1 \sigma \mathrm{rms} \sim 15 \mu \mathrm{Jy}$, and the depth of the Very Large Array (VLA) $8.2 \mathrm{GHz}$ map is $\sim 6 \mu \mathrm{Jy}$. $\mathrm{J} 163556$ is not detected in the two maps, placing $3 \sigma$ upper limits of $S_{1.4}<45 \mu \mathrm{Jy}$ and $S_{8.2}<18 \mu \mathrm{Jy}$.

\subsection{Optical and Near-infrared Imaging}

High angular resolution space-based optical and near-infrared observations have been obtained with the Advanced Camera for Surveys (ACS) and Near Infrared Camera and Multi-Object Spectrometer (NICMOS) on the Hubble Space Telescope (HST) for the programs 9292, 9452, 9717, 10325, and 10504 with a total exposure time in the range from 1.6 to $5.5 \mathrm{hr}$. The data reduction of these data is discussed in detail in Richard et al. (2008). In summary, the ACS data were reduced using the multidrizzle software (Koekemoer et al. 2002), which also removes cosmic rays and bad pixels, and combines the dithered frames to correct for camera distortions. The NICMOS data were reduced following the procedures given in the NICMOS data reduction handbook, then bad pixels were flagged, and subsequently using the LACOSMIC (van Dokkum 2001) IRAF procedures for cosmic-ray rejection.
Table 1

Photometry for J163556

\begin{tabular}{|c|c|c|c|}
\hline Telescope & $\lambda$ & Flux & R.A.(J2000) Decl.(J2000) \\
\hline WSRT & $1.4 \mathrm{GHz}$ & $<45 \mu \mathrm{Jy}$ & \\
\hline VLA & $8.2 \mathrm{GHz}$ & $<18 \mu \mathrm{Jy}$ & \\
\hline IRAM & $91 \mathrm{GHz}$ & $<0.44 \mathrm{mJy}$ & \\
\hline SMA & $870 \mu \mathrm{m}$ & $10.7 \pm 2.0 \mathrm{mJy}$ & $16: 35: 55.67+66: 12: 59.51$ \\
\hline JCMT & $850 \mu \mathrm{m}$ & $11.3 \pm 1.3 \mathrm{mJy}$ & $16: 35: 55.5+66: 13: 00$ \\
\hline JCMT & $450 \mu \mathrm{m}$ & $18.0 \pm 5.4 \mathrm{mJy}$ & \\
\hline Spitzer & $24.0 \mu \mathrm{m}$ & $<60 \mu \mathrm{Jy}$ & \\
\hline ISO & $15 \mu \mathrm{m}$ & $<150 \mu \mathrm{Jy}$ & \\
\hline Spitzer & $8.0 \mu \mathrm{m}$ & $22.03 \pm 0.10 \mathrm{mag}$ & \\
\hline ISO & $6.7 \mu \mathrm{m}$ & $<63 \mu \mathrm{Jy}$ & \\
\hline Spitzer & $5.8 \mu \mathrm{m}$ & $22.16 \pm 0.10 \mathrm{mag}$ & \\
\hline Spitzer & $4.5 \mu \mathrm{m}$ & $22.77 \pm 0.05 \mathrm{mag}$ & \\
\hline Spitzer & $3.6 \mu \mathrm{m}$ & $22.59 \pm 0.05 \mathrm{mag}$ & \\
\hline Subaru & K & $24.63 \pm 0.06 \mathrm{mag}$ & $16: 35: 55.64+66: 12: 59.32$ \\
\hline$H S T$ & F160W & $25.25 \pm 0.05 \mathrm{mag}$ & $16: 35: 55.66+66: 12: 59.45$ \\
\hline$H S T$ & F110W & $25.63 \pm 0.06 \mathrm{mag}$ & $16: 35: 55.64+66: 12: 59.37$ \\
\hline$H S T$ & F850LP & $25.69 \pm 0.05 \mathrm{mag}$ & $16: 35: 55.69+66: 12: 59.49$ \\
\hline$H S T$ & F775W & $26.03 \pm 0.05 \mathrm{mag}$ & $16: 35: 55.69+66: 12: 59.52$ \\
\hline$H S T$ & F625W & $27.55 \pm 0.13 \mathrm{mag}$ & $16: 35: 55.71+66: 12: 59.34$ \\
\hline$H S T$ & F555W & $>28.73 \mathrm{mag}$ & \\
\hline$H S T$ & F475W & $>29.55 \mathrm{mag}$ & \\
\hline$H S T$ & F435W & $>29.44 \mathrm{mag}$ & \\
\hline Chandra & $0.8-4 \mathrm{keV}$ & $\times 10^{-14} \mathrm{erg} \mathrm{s}^{-1}$ & \\
\hline
\end{tabular}

Ground-based observations in the $K$ band were obtained using Multi-Object InfraRed Camera and Spectrograph (MOIRCS) on Subaru (Ichikawa et al. 2006) as a part of the large search of extremely high-redshift lensed galaxies (Richard et al. 2008). The integration time is $\sim 5 \mathrm{hr}$ with good seeing conditions ( $\left(0^{\prime} \cdot 3-\right.$ 0 '.4) using dithered exposures of $50 \mathrm{~s}$ duration. The data were reduced using the MCSRED software package ${ }^{8}$ and the details on this are described in Richard et al. (2008).

The astrometry of the optical and near-infrared images was obtained using the USNO and the Two Micron All Sky Survey (2MASS) catalogs for the field, and the dispersion gives an accuracy $<0$ '.2.

Optical and near-infrared photometry were performed using SExtractor (Bertin \& Arnouts 1996) for a 0.5 arcsec aperture for the $H S T$ data and with a standard aperture correction in the $K$ band. When undetected, we place $3 \sigma$ upper limits. The photometry for the source $z 4$, which is at a distance of 0.6 arcsec from the SCUBA position, is given in Table 1, and all magnitudes are in the AB system (Oke 1974). The images are shown in Figure 1.

\subsection{Mid-infrared Imaging}

The ultra-deep (10 hr/band) Infrared Array Camera (IRAC) data of Abell 2218 were obtained as part of the Spitzer GO-2 program PID 20439 (PI: Eiichi Egami) while the deep ( 3000 s) MIPS $24 \mu \mathrm{m}$ data were taken as part of the Spitzer GTO program PID 83 (PI: George Rieke); see Egami et al. (2006) for the details of the IRAC and MIPS data reductions.

$\mathrm{J} 163556$ is detected in all four IRAC bands $(3.6,4.5,5.8$, and $8.0 \mu \mathrm{m}$ ) but not at $24 \mu \mathrm{m}$. A convolved $H S T$ image was used for determining the $H S T$-IRAC colors. The convolved $H S T$ image was also used for the astrometry registration. The MIPS nondetection sets a $3 \sigma$ upper limit of $<40 \mu \mathrm{Jy}$ at $24 \mu \mathrm{m}$. J163556 is not detected in the Infrared Space Observatory (ISO) ISOCAM

\footnotetext{
8 Available from http://www.naoj.org/staff/ichi/MCSRED/mcsred.html.
} 

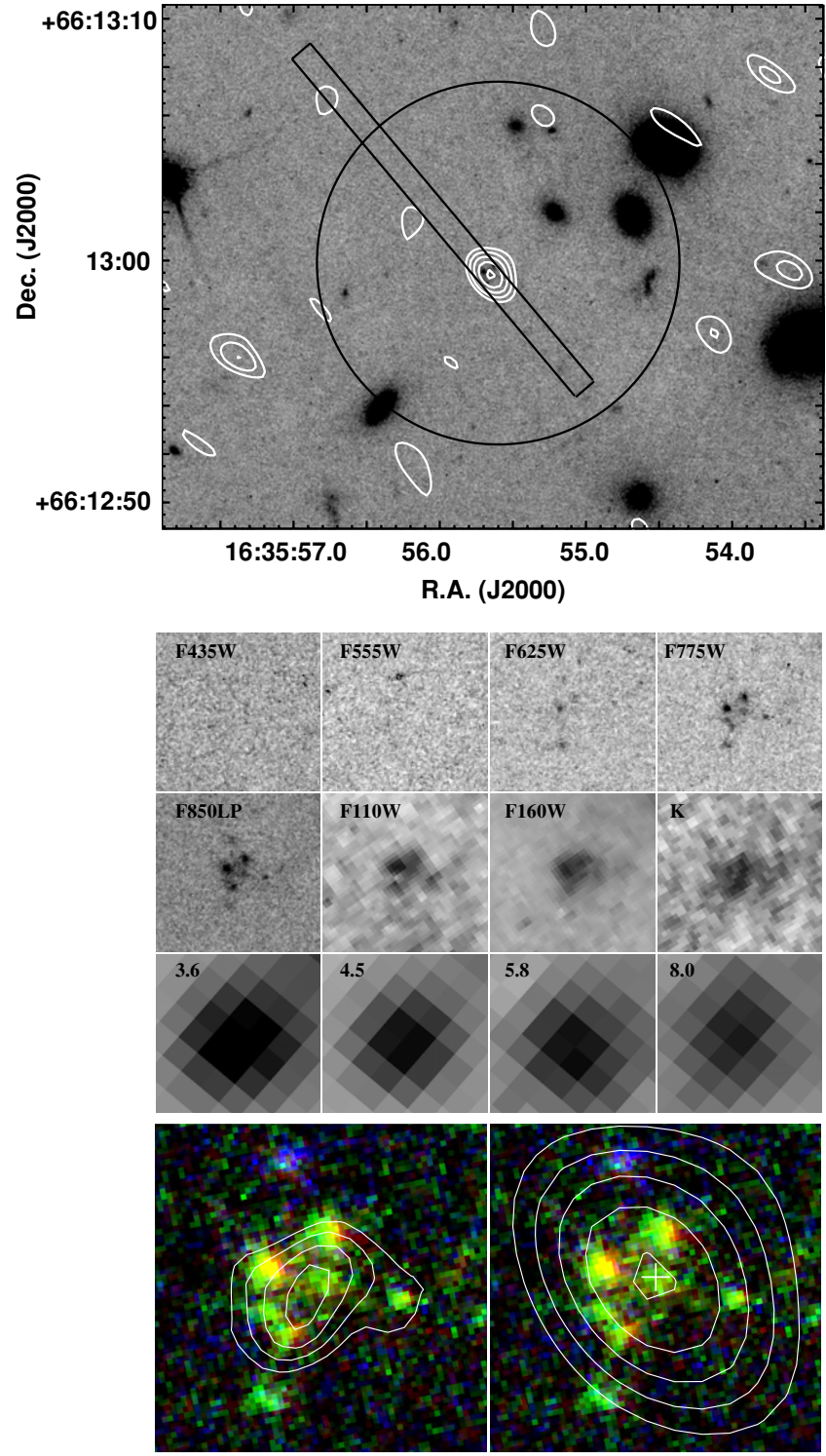

Figure 1. Top panel: F850LP $\left(z^{\prime}\right)$ image overlayed with the SMA contours (white; in steps of $1 \sigma$ rms. starting at $2 \sigma$ ). The black circle shows the SCUBA beam (FWHM of $\left.15^{\prime \prime}\right)$. The black rectangle shows the position of the slit used for optical spectroscopy. Middle panel: mosaic of the optical and near-infrared images of J163556. The size of each image is $\sim 4^{\prime \prime} \times 4^{\prime \prime}$. Bottom panel: is a $\sim 2^{\prime \prime} \times 2^{\prime \prime}$ true-color image of the F555, F775, and F850LP overlayed with left: $K$-band contours, and right: SMA contours, where the plus sign indicates the submm position as measured with the SMA (the plus is $0.2 \times 0.2$ ).

(A color version of this figure is available in the online journal.)

maps at 7 and $15 \mu \mathrm{m}$ (Metcalfe et al. 2003). We place $3 \sigma$ upper limits of 63 and $150 \mu \mathrm{m}$ Jy, respectively.

\subsection{Optical Spectroscopy}

We conducted deep multi-slit spectroscopy using the lowresolution imaging spectrograph (LRIS; mounted at the $10 \mathrm{~m}$ Keck Telescope on Mauna Kea, Hawaii; Oke et al. 1995) of sources in the rich galaxy cluster field A 2218. The data were obtained during the nights of 2003 June 30 and July 1, which had reasonable seeing, $\sim 0^{\prime} .8$, but were not fully photometric (with some cirrus). We obtained a crude flux calibration of our observations using Feige 67 and 110 as spectrophotometric standard stars. We observed the galaxy $z 4$ (see Section 2.3) for a total of $11.8 \mathrm{ks}$ using the $400 / 8500$ red grating and a total of
$10.6 \mathrm{ks}$ using the $600 / 4000$ blue gratings offering respectively a spectral dispersion of $0.63 \AA$ pixel $^{-1}$ in the blue and $1.86 \AA$ pixel $^{-1}$ in the red. No flux is detected in the blue channel below $5600 \AA$, where the dichroic splitter is located. We reduced these data that was part of a multi-object mask using standard IRAF scripts.

\section{RESULTS}

We fitted a point source to the SMA data to determine the position of the J163556. When fitting to the $u v$-data we find point source at the position R.A., decl. (J2000) = 16:35:55.67, 66:12:59.51 with positional errors of 0.15 arcsec with a flux of $10.7 \pm 2.0 \mathrm{mJy}$, and consistent results are found for the upper and lower sidebands, individually. When fitting to the synthesized map, we find R.A., decl. (J2000) = 16:35:55.64 \pm $0.02,+66: 12: 59.44 \pm 0.14$, and a flux of $10.7 \pm 0.2 \mathrm{mJy}$, with similar results obtained using routines JMFIT from AIPS and imfit from MIRIAD. We note that this position is only an arcsecond from the SCUBA position (Knudsen et al. 2008), demonstrating that good positions can be obtained for sources in the coarse resolution maps when the signal-to-noise ratio is sufficiently high (in this case $\sim 10$ ). The flux determined by the SMA data agrees with the SCUBA flux, further verifying the source is unresolved in the SMA image.

In Figure 1, we show the SMA data overlaid on the optical data. The multiwavelength photometry for the underlying counterpart galaxy of J163556 is presented in Table 1. The optical/ near-infrared position is determined in filter bands $\mathrm{F} 625 \mathrm{~W}$ to $K$. Because of the complex morphology of $z 4$, we smoothed the ACS images with a 0"'6 Gaussian kernel and the F110W band with a 0 '.5 Gaussian kernel before determining the position. The morphology varies between the different optical/near-infrared bands. We derive a mean optical/near-infrared position for $z 4$ of R.A., decl. $(\mathrm{J} 2000)=16: 35: 55.67 \pm 0.03,+66: 12: 59.42 \pm$ 0.08 . The offset between this mean position and the SMA position derived from the $u v$-data is $0^{\prime \prime} 09$, and the uncertainty on the offset is 0 '.23 in R.A. and $0^{\prime \prime} 17$ in decl.; hence we conclude that optical and submm position agree well.

Figure 2 shows the two-dimensional and one-dimensional extraction of this object within the 6000-6700 $\AA$ window, where we have identified an (asymmetric) emission line centered at $\lambda=6135 \AA$. Two independent reductions of the data were carried out producing similar results, and thus the emission line is most probably not spurious. A faint continuum is weakly visible redwards of the detected emission line. In light of other evidence discussed below, we believe this line to be Ly $\alpha$, which correspond to a redshift $z=4.044 \pm 0.001$, obtained through fitting to the emission line and a few absorption lines. We note these absorption lines are marginal and thus carry a low weight. A break in the continuum bluewards of the line as well as the lack of continuum emission in the blue part of the spectrum supports this identification. Alternatives at low redshift such as [O III] at $z=0.23$ or [O II] at $z=0.65$ seem less likely due to the absence of other lines and/or the lack of continuum on the blue side.

\section{DISCUSSION}

\subsection{Redshift}

The ratio of the $450 \mu \mathrm{m}$ and $850 \mu \mathrm{m}$ fluxes of 1.6 is strongly indicative of $\mathrm{J} 163556$ being a high-redshift source with $z \geqslant 3.5$, assuming that the far-infrared spectral energy distribution (SED) 


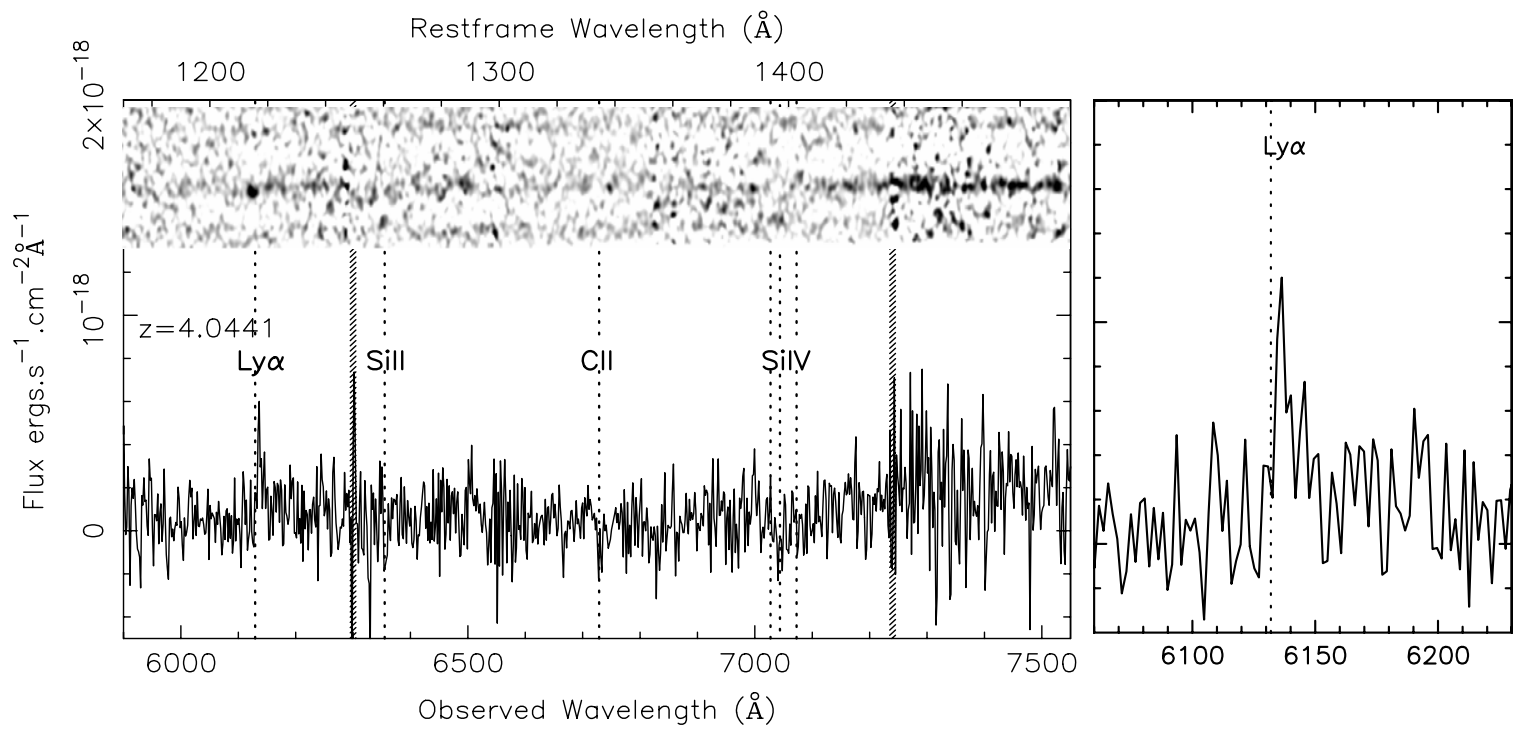

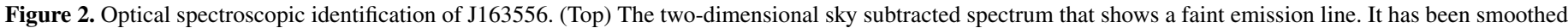

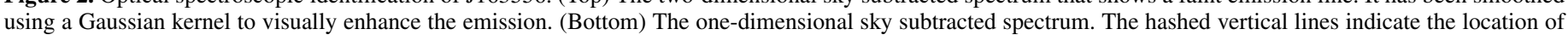

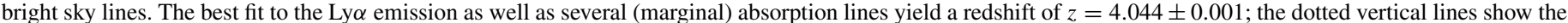
position of the Ly $\alpha$ line and the absorption lines. The right panel shows a zoom in on the Ly $\alpha$ line.

is well-described by a modified blackbody (as illustrated in Figure 3). A high redshift is supported by the $1.4 \mathrm{GHz}$ to $850 \mu \mathrm{m}$ flux ratio of $<0.004$, where the depth of the radio data allows a constraint of $z>3$. In Figure 3, we show how the $1.4 \mathrm{GHz}$ to $850 \mu \mathrm{m}$ flux density ratio changes with redshift using the relation from Carilli \& Yun (1999). As discussed in Blain (1999) and Carilli \& Yun (1999), the radio-submm flux can be used as a redshift indicator, assuming that both the radio and submm emission is powered by star formation. Additional evidence for $\mathrm{J} 163556$ being at high redshift $(z>3.5)$ comes from the $24 \mu \mathrm{m}$ to $850 \mu \mathrm{m}$ flux ratio $S_{24} / S_{850}<0.005$ (e.g., Figure 3, Pope et al. 2006). We note that the individual flux ratios in the radio, submm, and far-infrared, only provide crude redshift estimates, and are each known to have a large scatter (e.g., Carilli \& Yun 2000).

As the optical spectrum has only a single emission line, we calculate the photometric redshift using the Hyperz code (Bolzonella et al. 2000) and the photometry from the F435W $(=B)$ band to the IRAC bands. We see an excess in the $3.6 \mu \mathrm{m}$ band (shown in Figure 4), which is most probably caused by $\mathrm{H} \alpha$ emission (Yabe et al. 2009). For redshifts $z=3.84-5.03$ the $\mathrm{H} \alpha$ line will shift through the $3.6 \mu \mathrm{m}$ IRAC channel. We make a correction for the $\mathrm{H} \alpha$ emission deriving a line luminosity based on $10 \%$ of the star formation rate (SFR) assuming a typical FIR luminosity for SMGs. Excluding the $3.6 \mu \mathrm{m}$ data point, we find a photo- $z$ of 4.67 , while including the $\mathrm{H} \alpha$ corrected $3.6 \mu$ m data point we find a photo- $z$ of 4.77 . This high photometric redshift strongly supports our identification of the emission line in the optical spectrum as Ly $\alpha$ and not, e.g., low-redshift [O II].

Adopting a redshift of 4 , we estimate the gravitational lensing magnification using the cluster potential model from Elíasdóttir et al. (2007) as input to LENSTOOL (Jullo et al. 2007). We find a magnification factor of $5.5 \pm 1.9^{9}$, and note that this does

\footnotetext{
9 We note that this value, although different, is consistent with the magnification factor that we used in Knudsen et al. (2009). The difference is a consequence of a recent revision of the model in Elíasdóttir et al. (2007), which affects this region of the cluster, and the fact that in Knudsen et al. (2009) was determined for a single position, while here an extensive analysis to determine the uncertainty was carried out. Differences of $12 \%-20 \%$ are generally to be expected.
}
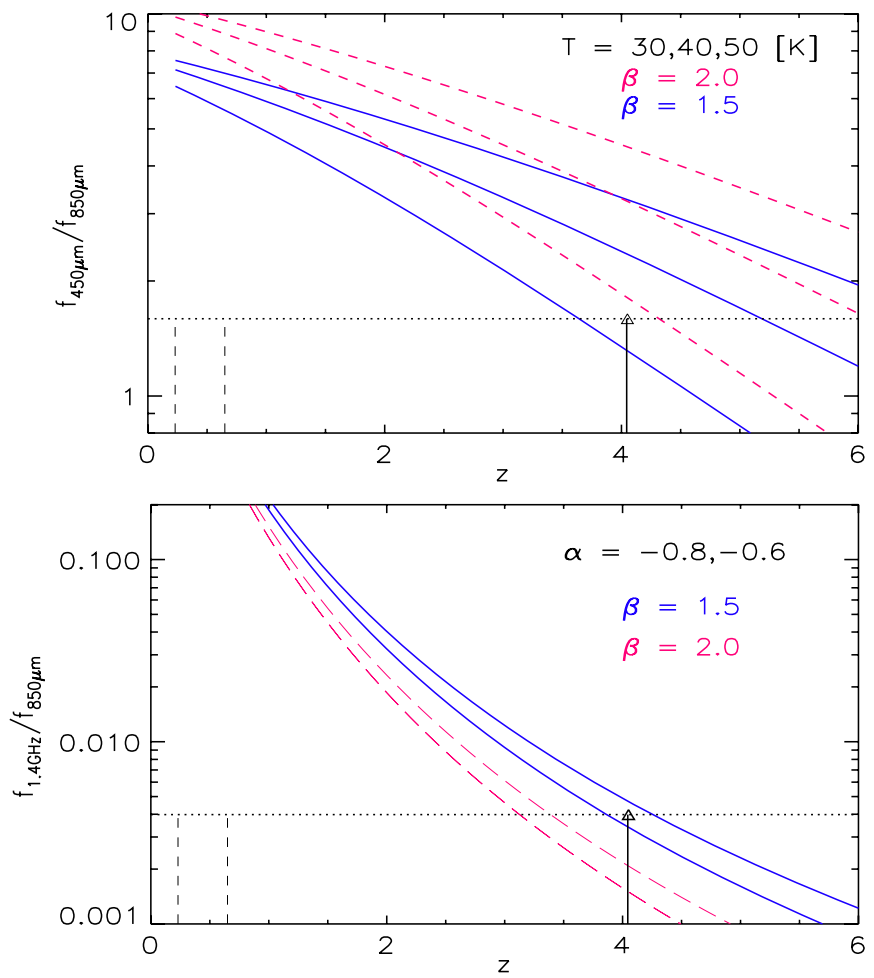

Figure 3. Predicted $450 \mu \mathrm{m}$ to $850 \mu \mathrm{m}$ ratio and $1.4 \mathrm{GHz}$ to $850 \mu \mathrm{m}$ flux ratio as function of redshift. The former is calculated assuming a modified blackbody SED (with $T$ going from 30 to $50 \mathrm{~K}$ from left to right), and the latter is based on the relation from Carilli \& Yun (1999), with $\alpha=-0.8$ as the left curve. The horizontal dotted line shows the flux ratio and flux ratio limit, respectively. The vertical solid line indicates $z=4.044$, while the vertical dashed lines show the redshift 0.23 and 0.65 .

(A color version of this figure is available in the online journal.)

not change much for redshift $z>2$. The spatial magnification is a factor of 3.2 in the direction tangential to the direction of the cluster center and 1.7 in the direction perpendicular. In Figure 5, we show the reconstruction of the source plane image of J163556 using the World Coordinate System (WCS) images. 


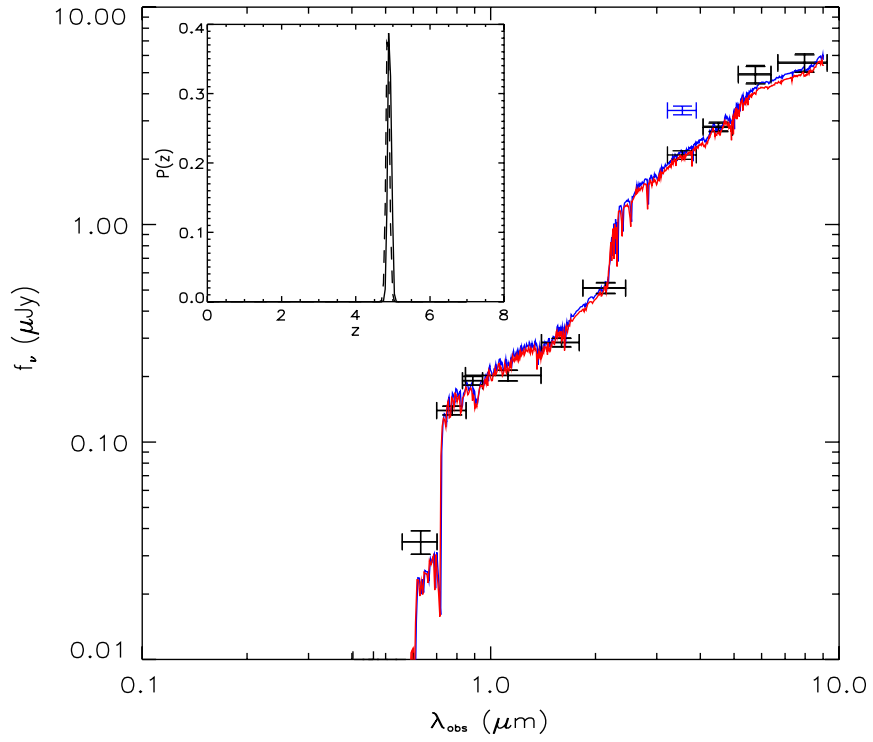

Figure 4. SED from the F435W to $8.0 \mu \mathrm{m}$ photometry overlayed with the bestfit spectrum from Hyperz from the photo- $z$ modeling of the optical counterpart of J163556. The blue $3.6 \mu \mathrm{m}$ shows the photometry as measured, and the black $3.6 \mu \mathrm{m}$ data point has been corrected for $\mathrm{H} \alpha$ emission line contribution. The blue spectrum shows the fit when including the $\mathrm{H} \alpha$ corrected $3.6 \mu \mathrm{m}$ data poin $\left(z_{\mathrm{ph}}=4.77\right)$ and the red spectrum shows the fit when excluding the $3.6 \mu \mathrm{m}$ photometry from the photo- $z$ estimate $\left(z_{\mathrm{ph}}=4.67\right)$. The inset shows the output probability distribution for the photometric redshift from Hyperz, where the solid line corresponds to the fit including the corrected $3.6 \mu \mathrm{m}$ photometry, and the dashed line corresponds to the fit with $3.6 \mu \mathrm{m}$ excluded.

(A color version of this figure is available in the online journal.)

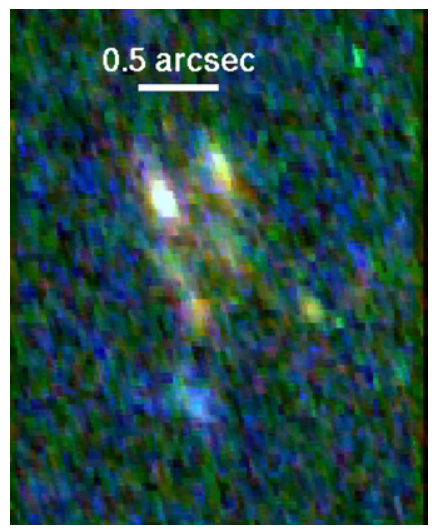

Figure 5. Reconstruction of the source plane image of J163556 shown using WCS images in the filters $625 \mathrm{~W}, 775 \mathrm{~W}$, and $850 \mathrm{LP}$.

(A color version of this figure is available in the online journal.)

The morphology of the object is only slightly distorted, albeit all the scales are reduced by a factor of $\sim 2$. The different colors of the clumps are likely due to different reddening.

The CO(4-3) line was not detected for J163556 in IRAM Plateau de Bure observations in the redshift range $z=$ 4.035-4.082 (Knudsen et al. 2009). Assuming a line width of $400 \mathrm{~km} \mathrm{~s}^{-1}$ Knudsen et al. (2009) placed an upper limit on the CO line luminosity of $0.3 \times 10^{10} \mathrm{~K} \mathrm{~km} \mathrm{~s}^{-1} \mathrm{pc}^{2}$ (using a lensing magnification of 5.5). This upper limit is not inconsistent with the $L_{\mathrm{CO}}^{\prime}-L_{\mathrm{FIR}}$ relation for starburst galaxies; ${ }^{10}$ hence the non-detection does not contradict the determined redshift $z=4.044$. It is important to keep in mind that we do not know

${ }^{10}$ We note that the far-infrared luminosity given in Knudsen et al. (2009) unfortunately missed a factor $(1+z)^{-1}$.

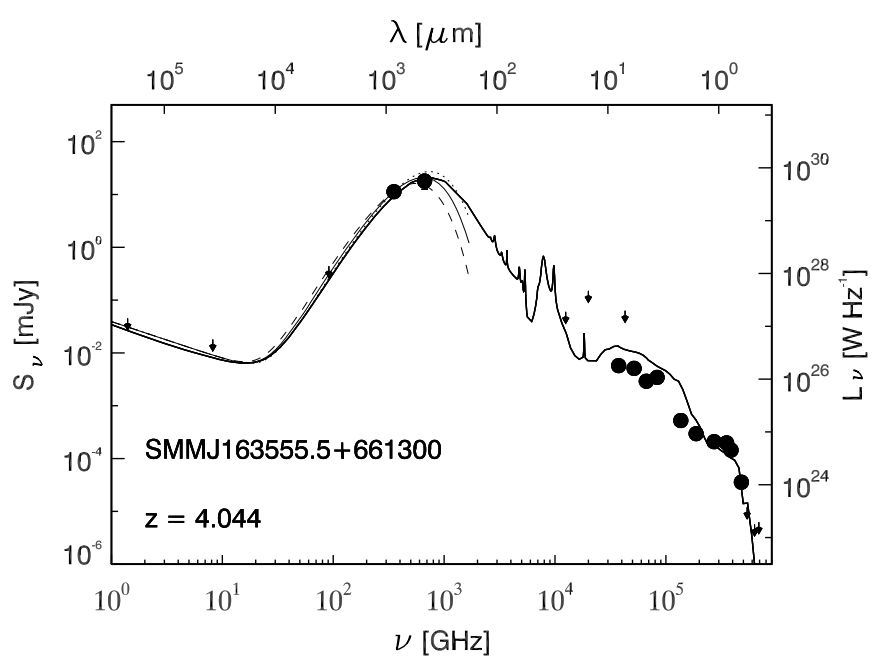

Figure 6. SED of J163556, overlayed with a modified blackbody with $T_{d}=$ $30,35,40 \mathrm{~K}$ (dashed, solid, dotted, respectively) and $\beta=1.5$, as well as the SED model for Arp220 from Silva et al. (1998) scaled to the submm points of J163556.

if the $\mathrm{CO}(4-3)$ transition is thermalized. While several bright SMGs have been found to have thermalized $\mathrm{CO}$ at least up to the (3-2) transition (e.g., Tacconi et al. 2008), cases of Milky Way type molecular gas excitation conditions have been found for a $z \sim 1.5 \mathrm{BzK}$ galaxy (Daddi et al. 2008; Dannerbauer et al. 2009) as well as subthermal excitation of the $\mathrm{CO}(2-1)$ and $\mathrm{CO}(5-4)$ in the submm bright extremely red object HR10 (Greve et al. 2003; Papadopoulos \& Ivison 2002). The CO(4-3) non-detection could also be explained by a velocity shift between the Ly $\alpha$ and the CO larger than $-525 \mathrm{~km} \mathrm{~s}^{-1}$, which is the difference between $z=4.035$ and 4.044 for $\mathrm{CO}(4-3)$. Shifts of $z_{\mathrm{Ly} \alpha}-z_{\mathrm{CO}}=-500 \mathrm{~km} \mathrm{~s}^{-1}$ have been seen for other SMGs (Greve et al. 2005).

\subsection{Spectral Energy Distribution}

Based on the $850 \mu \mathrm{m}$ and the $450 \mu \mathrm{m}$ data points, the far-infrared SED is well-described by a modified blackbody spectrum, with a dust temperature of $\sim 35 \mathrm{~K}$ assuming $\beta=1.5$, where $\beta$ is $f_{v} \propto v^{\beta}$. The dust temperature is comparable to what is seen in other SMGs at lower redshift (e.g., Kovács et al. 2006). If the single optical emission line would not be $\mathrm{Ly} \alpha$, but [O III] or [O II] implying a low redshift, the implied dust temperature would be 9-11 K. In Figure 6, we show the multiwavelength SED overlayed with a modified blackbody spectrum and the Arp220 SED from Silva et al. (1998). The far-infrared luminosity derived for this redshift 4.044 is $1.3_{-0.3}^{+0.7} \times 10^{12} L_{\odot}$ after correcting for gravitational lensing and where the two uncertainties represent the upper and lower limits from the magnification correction. J163556 is intrinsically a fainter object than the other $z>4$ SMGs, which have estimated FIR luminosities $\sim 10^{13} L_{\odot}$ (Capak et al. 2008; Daddi et al. 2009a, 2009b; Coppin et al. 2009). Assuming a Salpeter initial mass function, solar abundance, a continuous burst for 10-100 Myr, and that the dust reradiates all the bolometric luminosity, the far-infrared luminosity implies an SFR of $\sim 230 M_{\odot} \mathrm{yr}^{-1}$ (Kennicutt 1998).

Assuming that the submm and radio emission from J163556 are correlated, we use the radio-submm flux ratio as a function of redshift as discussed by Blain (1999) to estimate the radio flux. We estimate $S_{1.4} \sim 40 \mu \mathrm{Jy}$. Assuming that the radio SED is described by a power law, $S_{v} \propto v^{\alpha}$ with $\alpha=-0.7$, then 
$S_{8.2} \sim 13 \mu \mathrm{Jy}$. Both estimated fluxes are below the sensitivity levels of the WSRT and VLA maps. This implies that no radioloud active galactic nucleus (AGN) is present, and thus the submm and radio emission are most likely powered by star formation rather than AGN activity. The absence of a dominating AGN or quasar is also supported by the lack of an X-ray detection in the Chandra observations (Machacek et al. 2002; Govoni et al. 2004); in the $0.8-4 \mathrm{keV}$ band, an upper limit of $1.24 \times 10^{-14} \mathrm{erg} \mathrm{s}^{-1} \mathrm{~cm}^{-2}$ is estimated for an area of $3 \times 3$ pixels. We note that this is the first $z>4$ SMG identification without radio counterpart, although we also point out that the radio observations are not as deep as those used for the identification of other $z>4$ SMGs.

To determine the properties of J163556 we use Hyperz and find that the optical and near-infrared photometry is best fit by a star formation history of an elliptical galaxy template with an age of $\sim 1.4 \mathrm{Gyr}$ and a reddening of $A_{V}=0.8 \mathrm{mag}$. The rest-frame $K$-band absolute magnitude is -24.40 . For this template, the $K$-band luminosity would correspond to a stellar mass of $8.7 \times 10^{10} M_{\odot}$ without correcting for the gravitational lensing. Correcting for lensing magnification this corresponds to $\sim 1.6 \times 10^{10} M_{\odot}$. The results of the fit, however, yield an age close to the age of the universe at that redshift, $\sim 1.5 \mathrm{Gyr}$. Additionally, we fix the redshift to the spectroscopic redshift and use different star formation histories from a single burst to a continuous star formation. A single burst gives the best fit with a reduced $\chi^{2}$ of 1.39 , a reddening of $A_{V}=1.8 \mathrm{mag}$, and a very young age of 10-20 Myr. Other star formation histories with an exponential decay or with continuous star formation yield a $\chi^{2}$ of 1.76, a reddening of $A_{V}=2.0 \mathrm{mag}$, and a best-fit age of 40 Myr.

We note that the Spitzer IRAC observations probe the light at rest-frame wavelengths of $0.71-1.6 \mu \mathrm{m}$, which for an elliptical galaxy is dominated by the evolved stellar populations. However, for a starburst galaxy this could be dominated by young supergiants, as is seen in local ULIRGs. It is known that there is a degeneracy between a mature stellar population with small or modest amount of extinction and a younger population with a much stronger extinction. A younger population would imply a lower stellar mass estimate and thus the best-fit estimate can be considered an upper limit.

Based on the F775W filter, we can compute the rest-frame UV continuum luminosity $L(1500 \AA)=2.0 \times 10^{22} \mathrm{~W} \mathrm{~Hz}^{-1}$ and derive an unlensed SFR of $\sim 28 M_{\odot} \mathrm{yr}^{-1}$ using the Kennicutt (1998) empirical calibration (although certainly underestimated due to dust extinction). This result compared to the infrared luminosity suggests that the vast majority of the young stars in this galaxy are obscured by dust and are undetectable in the rest-frame far-UV.

\subsection{Size and Morphology}

The HST images give the best insight into the morphology of the galaxy. The resolution is the highest in the ACS F850LP $\left(z^{\prime}-\right.$ band) image, which probes redshifted UV light (see Figure 1). The galaxy is seen as a very irregular system made of four knots. The total extent of the galaxy in the $z^{\prime}$-band image is $1^{\prime \prime} .8 \times 1^{\prime \prime}$. 1 . Correcting for the lensing magnification, the source size is then $0{ }^{\prime} 9 \times 0$ 0' 4 . For $z=4.044$ this corresponds to a physical scale of $\sim 6 \mathrm{kpc} \times 3 \mathrm{kpc}$. The largest star-forming knot is unresolved in the ACS images, and we place an upper limit on the extent of about $0^{\prime \prime} 07$ or $500 \mathrm{pc}$ in the source plane. In the $K$-band (which corresponds to rest-frame $B$-band) image, the light originates primarily from the central part of the galaxy. We suggest

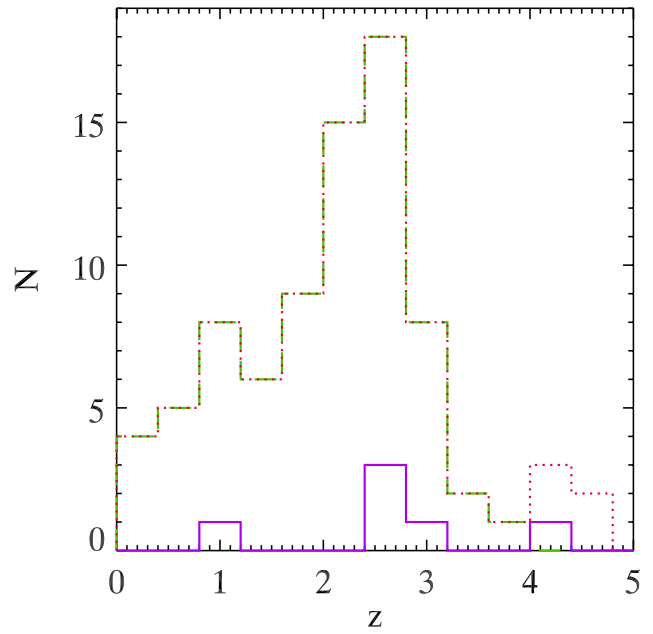

Figure 7. Redshift distribution for faint and bright SMGs. The solid (purple) histogram shows the redshift distribution of the reliably identified lensed SMGs with $S_{850 \mu \mathrm{m}}<2 \mathrm{mJy}$. The long dashed (green) histogram shows the redshift distribution from Chapman et al. (2005), and the dotted (red) histogram shows the same including the five redshifts from Capak et al. (2008), Coppin et al. (2009), Daddi et al. 2009a, 2009b).

(A color version of this figure is available in the online journal.)

that there are already old stars in place at the center of this galaxy.

J163556 is detected as a point source in the SMA data. Correcting the beam shape for a gravitational lensing amplification of $3.2 \times 1.7$ in the tangential and the radial direction, respectively, of the cluster potential well, we find the beam to be $0^{\prime \prime} .47 \times 1^{\prime \prime} .2$ in the source plane. This is equivalent to the resolution that can be obtained with the SMA in extended configuration without gravitational lensing. Through a two-dimensional Gaussian fit to the SMA data we are not able to resolve the source concluding that it is point-like within the SMA beam. We place a strict upper limit on the size of the starburst region using the lensing corrected beam. In the $z=4.044$ source plane, this corresponds to $8 \mathrm{kpc} \times 3 \mathrm{kpc}$. For an SFR of $\sim 230 M_{\odot} \mathrm{yr}^{-1}$, this yields a lower limit for the SFR density of $12 M_{\odot} \mathrm{yr}^{-1} \mathrm{kpc}^{-2}$.

\subsection{Extending the Redshift Distribution of Faint SMGs}

The previously identified five $z>4$ bright SMGs have farinfrared luminosities 5-10 times larger than J163556. For the six other reliably identified faint SMGs with $S_{850 \mu \mathrm{m}}<2 \mathrm{mJy}$ (Frayer et al. 2003; Kneib et al. 2004; Borys et al. 2004; Knudsen et al. 2009; K. K. Knudsen et al. 2010, in preparation), the redshifts range from 1.03 to 2.9 with a majority of the sources found around $z \sim 2.5$. Due to the modest number of known faint SMGs, their redshift distribution is poorly constrained, and it has been proposed by several groups that the faint SMGs or lowluminosity SMGs peak at lower redshifts than the bright SMGs (e.g., Wall et al. 2008). Our result for J163556 thus extends the redshift distribution of faint SMGs to very high redshifts.

In Figure 7, we plot the redshift distribution of $S_{850 \mu \mathrm{m}}<$ 2 mJy SMGs with spectroscopic redshifts in comparison to the redshift distribution for radio-identified, blank field SMGs (Chapman et al. 2005). Between redshift 1 and 8, due to the negative $k$-correction in the submm, the observed flux density stays essentially constant for a given far-infrared luminosity. As several SMGs have their far-infrared luminosity determined from single submm fluxes, we use the flux density rather than the luminosity for our simple sample comparison. As the 
radio-identified SMGs are expected to represent the general SMG population, but with a possible bias against redshifts $z>3.5$, we also include the five $z>4$ bright SMGs (Capak et al. 2008; Coppin et al. 2009; Daddi et al. 2009a, 2009b). Using the non-parametric rank sum test known as the Mann-Whitney $U$-test (e.g., Babu \& Feigelson 1996), we find that there is only a $1.3 \sigma(1.6 \sigma)$ level of significance that the faint SMGs have a different redshift distribution than the Chapman et al. sample + the $z>4$ SMGs (the Chapman et al. sample alone). Removing the $z<1$ tail of the Chapman et al. sample, as the negative $k$-correction does not apply in that redshift range, we find a consistent result. In other words, based on this small number of faint SMGs we find that it is possible that the less luminous SMGs have a similar redshift distribution as the more luminous SMGs.

With the model predictions for the redshift distribution of SMGs (e.g., Chapman et al. 2005; Wall et al. 2008) in most submm surveys (e.g., Smail et al. 2002; Webb et al. 2003; Coppin et al. 2006; Knudsen et al. 2008) at least a few sources are expected to have a $z>4$. As $z \sim 2$ massive, old evolved galaxies $\left(>10^{11} M_{\odot},>2 \mathrm{Gyr}\right)$ are observed with a space density of $\sim 10^{-4} \mathrm{Mpc}^{-3}$ (e.g., Daddi et al. 2005; Kong et al. 2006), these galaxies probably had their dominant stellar population formed in a single starburst at redshift $z>4$ (e.g., Daddi et al. 2005; Stockton et al. 2008). The most likely progenitor of these are the massive starbursts that we observe in SMGs. If the large SFRs of $\sim 1000 M_{\odot} \mathrm{yr}^{-1}$ can be sustained over a period of $\sim 100 \mathrm{Myr}$, it is possible to build such large stellar masses (e.g., Coppin et al. 2009). Thus identification of $\geqslant 4$ SMGs is vital for our understanding of $z \sim 2$ massive, old galaxies.

To reliably identify $z>4$ SMGs, deep data at all wavelengths are necessary. Though the strongest, initial probe comes from the ratio between $450 \mu \mathrm{m}$ and $850 \mu \mathrm{m}$ fluxes, or possibly $350 \mu \mathrm{m}$ and $850 \mu \mathrm{m}$, where this ratio is expected to be $<2$. In most cases, this requires very deep $450 \mu \mathrm{m}$ or $350 \mu \mathrm{m}$ observations with a $1 \sigma \mathrm{rms}<5 \mathrm{mJy}$ beam $^{-1}$. Thus the possibilities for systematic searches for very high-redshift SMGs rely on the success of the future high-sensitive submm cameras such as SCUBA-2 and space telescopes such as Herschel to provide such observations.

\section{CONCLUSIONS}

We report the identification of the gravitationally lensed SMG SMMJ163555.5+661300 (J163556), which has a lensing corrected far-infrared luminosity of $1.3 \times 10^{12} L_{\odot}$. Using SMA interferometric observations, we have identified the underlying optical counterpart. The SMA flux agrees well with the SCUBA flux of $11.3 \mathrm{mJy}$.

1. From optical spectroscopy from the Keck Telescope, we detect an asymmetric emission line at $6135 \AA$ and no emission on the blue side. We identify this emission line as the Ly $\alpha$ line, which implies a redshift of $4.0441 \pm 0.001$. Using photometric redshift calculations, we rule out that the single emission line could be [O II] or [O III], which would otherwise have implied a redshift of 0.25 and 0.61 , respectively. This is also strongly supported by the flux ratios at submm and radio wavelengths.

2. J163556 is not resolved in the SMA data, and we place a strict upper limit on the starburst size of $8 \mathrm{kpc} \times 3 \mathrm{kpc}$. From the derived SFR of $230 M_{\odot} \mathrm{yr}^{-1}$ we estimate a lower limit on the SFR surface density of $12 M_{\odot} \mathrm{yr}^{-1} \mathrm{kpc}^{-2}$.

3 . With an unlensed flux of $\sim 2 \mathrm{mJy}, \mathrm{J} 163556$ is fainter than the classical (bright) SMGs that are often classified at
$S_{850 \mu \mathrm{m}}>5 \mathrm{mJy}$. It is thus so far the most distant known faint SMG. This result extents the redshift distribution of the faint SMGs that are typically only found in gravitational lensing surveys to look back times equivalent to the bright SMGs.

4. The derived upper limit for the $\mathrm{CO}(4-3)$ line luminosity of $0.3 \times 10^{10} \mathrm{~K} \mathrm{~km} \mathrm{~s}^{-1} \mathrm{pc}^{-2}$ is not inconsistent with the $L_{\mathrm{CO}}^{\prime}-L_{\mathrm{FIR}}$ relation for starburst galaxies. The $\mathrm{CO}(4-3)$ nondetection is possibly caused by subthermal excitation or a large velocity shift.

5. Based on the small number of reliably identified faint, lensed SMGs with spectroscopic redshift, we do not find evidence that they have a different redshift distribution than that of bright SMGs as traced by the radio-identified SMGs from Chapman et al. (2005).

We thank an anonymous referee for insightful comments, which help improve the manuscript. The authors recognize and acknowledge the very significant cultural role and reverence that the summit of Mauna Kea has always had within the indigenous Hawaiian community. We are most fortunate to have the opportunity to conduct observations from this mountain. This program is based on observations made with the NASA/ESA Hubble Space Telescope, which is operated by the Association of Universities for Research in Astronomy, Inc., under NASA contract NAS 5-26555, the Subaru Telescope, which is operated by the National Astronomical Observatory of Japan, the Spitzer Space Telescope, which is operated by the Jet Propulsion Laboratory, California Institute of Technology under a contract with NASA. The Submillimeter Array is a joint project between the Smithsonian Astrophysical Observatory and the Academia Sinica Institute of Astronomy and Astrophysics and is funded by the Smithsonian Institution and the Academia Sinica. We thank Federica Govoni and Marie Machacek for providing the Chandra X-ray upper limit. K.K. acknowledges support from the Deutsche Forschungsgemeinschaft (DFG) Priority Programme 1177. J.P.K. thanks CNRS for support. J.R. acknowledges support from a EU Marie-Curie fellowship.

Facilities: SMA, Keck (LRIS), JCMT (SCUBA), HST (ACS, NICMOS), Spitzer (IRAC, MIPS), WSRT, VLA, Subaru (MOIRCS)

\section{REFERENCES}

Babu, G. J., \& Feigelson, E. D. (ed.) 1996, Astrostatistics (London: Chapman and Hall)

Bertin, E., \& Arnouts, S. 1996, A\&AS, 117, 393

Blain, A. W. 1999, MNRAS, 309, 955

Bolzonella, M., Miralles, J.-M., \& Pelló, R. 2000, A\&A, 363, 476

Borys, C., et al. 2004, MNRAS, 352, 759

Capak, P., et al. 2008, ApJ, 681, L53

Carilli, C. L., \& Yun, M. S. 1999, ApJ, 513, L13

Carilli, C. L., \& Yun, M. S. 2000, ApJ, 530, 618

Chapman, S. C., Blain, A. W., Ivison, R. J., \& Smail, I. R. 2003, Nature, 422, 695

Chapman, S. C., Blain, A. W., Smail, I., \& Ivison, R. J. 2005, ApJ, 622, 772

Coppin, K., et al. 2006, MNRAS, 372, 1621

Coppin, K. E. K., et al. 2009, MNRAS, 395, 1905

Daddi, E., Dannerbauer, H., Elbaz, D., Dickinson, M., Morrison, G., Stern, D., \& Ravindranath, S. 2008, ApJ, 673, L21

Daddi, E., Dannerbauer, H., Krips, M., Walter, F., Dickinson, M., Elbaz, D., \& Morrison, G. E. 2009a, ApJ, 695, L176

Daddi, E., et al. 2005, ApJ, 626, 680

Daddi, E., et al. 2009b, ApJ, 694, 1517

Dannerbauer, H., Daddi, E., Riechers, D. A., Walter, F., Carilli, C. L., Dickinson, M., Elbaz, D., \& Morrison, G. E. 2009, ApJ, 698, L178 
Dannerbauer, H., Lehnert, M. D., Lutz, D., Tacconi, L., Bertoldi, F., Carilli, C., Genzel, R., \& Menten, K. 2002, ApJ, 573, 473

Dannerbauer, H., Lehnert, M. D., Lutz, D., Tacconi, L., Bertoldi, F., Carilli, C., Genzel, R., \& Menten, K. M. 2004, ApJ, 606, 664

Dannerbauer, H., Walter, F., \& Morrison, G. 2008, ApJ, 673, L127

Egami, E., et al. 2006, ApJ, 647, 922

Elíasdóttir, Á., et al. 2007, arXiv:0710.5636

Frayer, D. T., Armus, L., Scoville, N. Z., Blain, A. W., Reddy, N. A., Ivison, R. J., \& Smail, I. 2003, AJ, 126, 73

Garrett, M. A., Knudsen, K. K., \& van der Werf, P. P. 2005, A\&A, 431, L21

Govoni, F., Markevitch, M., Vikhlinin, A., VanSpeybroeck, L., Feretti, L., \& Giovannini, G. 2004, ApJ, 605, 695

Greve, T. R., Ivison, R. J., \& Papadopoulos, P. P. 2003, ApJ, 599, 839

Greve, T. R., et al. 2005, MNRAS, 359, 1165

Ho, P. T. P., Moran, J. M. \& Lo, K. Y. 2004, ApJ, 616, L1

Holland, W. S., et al. 1999, MNRAS, 303, 659

Ichikawa, T., et al. 2006, Proc. SPIE, 6269, 38

Ivison, R. J., et al. 2002, MNRAS, 337, 1

Jullo, E., Kneib, J.-P., Limousin, M., Elíasdóttir, Á., Marshall, P. J., \& Verdugo, T. 2007, New J. Phys., 9, 447

Kennicutt, R. C., Jr. 1998, ARA\&A, 36, 189

Kneib, J.-P., van der Werf, P. P., Knudsen, K. K., Smail, I., Blain, A., Frayer, D., Barnard, V., \& Ivison, R. 2004, MNRAS, 349, 1211

Knudsen, K. K., Neri, R., Kneib, J.-P., \& van der Werf, P. P. 2009, A\&A, 496, 45

Knudsen, K. K., van der Werf, P. P., \& Kneib, J.-P. 2008, MNRAS, 384, 1611

Knudsen, K. K., et al. 2006, MNRAS, 368, 487

Koekemoer, A. M., Fruchter, A. S., Hook, R. N., \& Hack, W. 2002, in The 2002 HST Calibration Workshop: Hubble after the Installation of the ACS and the NICMOS Cooling System, ed. S. Arribas, A. Koekemoer, \& B. Whitmore (Baltimore, MD: Space Telescope Science Institute), 337
Kong, X., et al. 2006, ApJ, 638, 72

Kovács, A., Chapman, S. C., Dowell, C. D., Blain, A. W., Ivison, R. J., Smail, I., \& Phillips, T. G. 2006, ApJ, 650, 592

Machacek, M. E., Bautz, M. W., Canizares, C., \& Garmire, G. P. 2002, ApJ, 567,188

Metcalfe, L., et al. 2003, A\&A, 407, 791

Oke, J. B. 1974, ApJS, 27, 21

Oke, J. B., et al. 1995, PASP, 107, 375

Papadopoulos, P. P., \& Ivison, R. J. 2002, ApJ, 564, L9

Pope, A., et al. 2006, MNRAS, 370, 1185

Richard, J., Stark, D. P., Ellis, R. S., George, M. R., Egami, E., Kneib, J.-P., \& Smith, G. P. 2008, ApJ, 685, 705

Sault, R. J., Teuben, P. J., \& Wright, M. C. H. 1995, in ASP Conf. Ser. 77 , Astronomical Data Analysis Software and Systems IV, ed. R. A. Shaw, H. E. Payne, \& J. J. E. Hayes (San Francisco, CA: ASP), 433

Schinnerer, E., et al. 2008, ApJ, 689, L5

Silva, L., Granato, G. L., Bressan, A.. \& Danese, L. 1998, ApJ, 509, 103

Smail, I., Ivison, R. J., Blain, A. W., \& Kneib, J.-P. 2002, MNRAS, 331, 495

Stockton, A., McGrath, E., Canalizo, G., Iye, M., \& Maihara, T. 2008, ApJ, 672, 146

Tacconi, L. J., et al. 2008, ApJ, 680, 246

van Dokkum, P. G. 2001, PASP, 113, 1420

Wall, J. V., Pope, A., \& Scott, D. 2008, MNRAS, 383, 435

Wang, W.-H., Barger, A. J., \& Cowie, L. L. 2009, ApJ, 690, 319

Wang, W.-H., Cowie, L. L., van Saders, J., Barger, A. J., \& Williams, J. P. 2007, ApJ, 670, L89

Webb, T. M., et al. 2003, ApJ, 587, 41

Yabe, K., Ohta, K., Iwata, I., Sawicki, M., Tamura, N., Akiyama, M., \& Aoki, K. 2009, ApJ, 693, 507

Younger, J. D., et al. 2007, ApJ, 671, 1531 